Meta

Journal des traducteurs

Translators' Journal

\title{
Le foisonnement en traduction technique d'anglais en français
}

\section{Christine Durieux}

Volume 35, numéro 1, mars 1990

Actes du colloque international " La traduction proligère "

URI : https://id.erudit.org/iderudit/002689ar

DOI : https://doi.org/10.7202/002689ar

Aller au sommaire du numéro

Éditeur(s)

Les Presses de l'Université de Montréal

ISSN

0026-0452 (imprimé)

1492-1421 (numérique)

Découvrir la revue

Citer cet article

Durieux, C. (1990). Le foisonnement en traduction technique d'anglais en

français. Meta, 35(1), 55-60. https://doi.org/10.7202/002689ar d'utilisation que vous pouvez consulter en ligne.

https://apropos.erudit.org/fr/usagers/politique-dutilisation/ 


\section{LE FOISONNEMENT EN TRADUCTION TECHNIQUE D'ANGLAIS EN FRANÇAIS}

CHRISTINE DURIEUX

Université de la Sorbonne Nouvelle-Paris III, Paris, France

Avant de voir quelles sont les causes théoriques et méthodologiques du foisonnement et d'envisager des solutions, peut-être est-il utile de définir l'objet de notre propos.

Qu'est-ce que le foisonnement? Quelle que soit la langue de spécialité dans laquelle il apparaît, des travaux publics à la pâtisserie, ce terme désigne toujours un accroissement de volume. En traduction, le foisonnement est la proligération de mots en surnombre, c'est l'augmentation de volume du texte d'arrivée par rapport au texte de départ.

\section{CONSTAT}

Tout d'abord, un constat s'impose. Le foisonnement existe. Il est reconnu, il est même institutionnalisé et quantifié. En effet, un organisme professionnel comme la Société française des traducteurs (SFT) recommande aux traducteurs d'en tenir compte dans l'établissement de leurs devis. Cet organisme indique un coefficient de foisonnement, c'est-à-dire la différence de volume entre le texte original et le texte traduit, pour les langues européennes les plus courantes. Ainsi, pour la traduction d'anglais en français, qui est l'objet de notre étude, la SFT annonce un coefficient de foisonnement courant de 25 pour cent.

Ce calcul est fondé sur une analyse statistique portant sur de nombreuses pages de traduction. Néanmoins, on peut s'interroger sur la valeur numérique de ce coefficient, voire sur son bien-fondé. S'il s'agit de protéger le traducteur professionnel contre un donneur d'ouvrage exigeant et de prendre une marge de sécurité en prévoyant au départ, sur le plan tant de la rémunération que des délais, un volume de travail surestimé pour ne pas risquer de mauvaise surprise et pour être sûr de rester en fin de compte à l'intérieur de l'enveloppe de son devis, ce peut être une précaution prudente, mais le foisonnement n'est plus alors qu'un prétexte. S'il se justifie à la rigueur sur le plan socio-professionnel, le coefficient de foisonnement n'est en revanche guère fondé sur le plan traductologique. D'une part, ce coefficient de 25 pour cent dans le passage de l'anglais au français est fondé sur l'observation de traductions sur lesquelles aucun jugement de valeur n'est porté et, par conséquent, dont la qualité n'est aucunement garantie. Or, si la matière observée ne répond pas à des critères stricts de fidélité à l'information contenue dans le texte original et de qualité d'expression dans la langue d'arrivée, les résultats de l'analyse risquent d'être faussés. D'autre part, aucun fondement théorique ne justifie qu'il faille en français 25 pour cent de mots de plus qu'en anglais pour formuler un même énoncé. En outre, curieusement, on remarque que ce même organisme professionnel ne se prononce pas sur le corollaire. En effet, la SFT n'indique pas de coefficient négatif pour le passage du français à l'anglais.

Une première explication d'ordre général se dessine : quand on traduit, on manie la pensée d'autrui et, de ce fait, on a tendance à être plus explicite. En effet, on découvre des phénomènes, des arguments et des relations entre des idées et on pense logiquement qu'il 
faut expliciter l'implicite que l'on a perçu dans le texte original pour permettre au lecteur final d'accéder à la totalité du message. En revanche, quand on s'exprime spontanément dans sa langue maternelle, on est plus concis parce qu'on a tout l'implicite à l'esprit et que celui-ci semble tellement évident qu'il est inutile de le préciser.

\section{CAUSES}

Les principales causes du foisonnement procèdent en fait de défauts de méthode. Nous évoquerons rapidement les cinq causes les plus fréquentes.

La première cause est la surtraduction. De fait, la translation d'une langue à une autre s'accompagne souvent du passage d'un cadre culturel à un autre. Mais le traducteur doit-il pour autant expliquer ce changement de cadre? Par exemple, dans un document traitant du commerce international de produits agro-alimentaires, il est fait état à la rubrique des viandes d'une forte demande de dindes aux États-Unis en novembre pour le Thanksgiving Day. Dans ce contexte, il est inutile de préciser dans la traduction l'origine de cette fête et sa date habituelle. Même si le lecteur européen ignore ce qu'est le Thanksgiving Day, il se doute qu'il doit s'agir d'une fête - ne sert-on pas en France de la dinde à des repas de fête comme Noël —, que cette fête doit être propre aux États-Unis et qu'elle doit se situer fin novembre. Cette compréhension intuitive suffit au destinataire pour appréhender le sens de l'information fournie. Le traducteur $n$ 'a pas besoin de surtraduire, c'est-à-dire d'apporter des explications supplémentaires. Dans ce type de cas, le traducteur est souvent tenté d'étaler ses connaissances biculturelles et d'ajouter, en apposition après Thanksgiving Day, par exemple «fête américaine commémorant la journée d'action de grâce des pionniers après leur première récolte».

La deuxième cause est le pléonasme. Ce dont il est question ici, ce n'est pas la redondance linguistique fautive comme dans l'expression «monter en haut» ou «descendre en bas», mais la redondance dans l'information fournie. Par exemple, dans un rapport traitant de l'angioplastic par laser, on lit que l'un des risques majeurs de l'opération est de :

(...) produce particles that could clog smaller capillaries.

Cette phrase a été traduite par:

(...) détacher des particules qui risquent de boucher des petits capillaires.

Or, par définition, les capillaires sont de petits vaisseaux plus petits que les artères dans lesquels on pratique l'angioplastie par laser. Il aurait donc suffi d'écrire:

(...) qui risquent de boucher des capillaires.

La troisième cause est le calque. Le traducteur cherche à tout traduire, l'inflation verbale devient vite galopante. Prenons un autre extrait de ce même rapport:

Surgeons could soon be using lasers to look at material blocking clogged arteries during an operation.

Les chirurgiens pourraient bientôt utiliser des lasers pour visualiser la matière qui bloque les artères obstruées au cours d'une opération.

Les chiffres parlent d'eux-mêmes : 16 mots en anglais, 20 mots en français, soit 25 pour cent de plus.

L'inadéquation du calque comme méthode de traduction apparaît immédiatement avec l'ambiguité créée par le syntagme: «au cours d'une opération». De toute évidence, 
une restructuration de la phrase s'impose, mais même en plaçant «au cours d'une opération» plus tôt dans la phrase - «les chirurgiens pourraient bientôt utiliser des lasers en cours d'opération pour visualiser (...)» - on ne change rien au coefficient de foisonnement. En revanche, une formulation spontanée résultant de l'application de la méthode interprétative de la traduction ne donne que 16 mots, comme en anglais: «Des lasers devraient bientôt permettre d'étudier en cours d'intervention la matière qui obstrue l'artère.»

La quatrième cause est la prise en compte de la totalité du sémantisme d'un terme. Le traducteur allonge le syntagme pour tenter de couvrir toute la surface conceptuelle d'un terme. Par exemple :

Today, clogged coronary arteries are treated (...) by threading a balloon through the obstruction, then inflating it to push the artery open.

Dans cet extrait, nous retiendrons deux exemples pour illustrer notre propos: «threading (...) through et push open». Pour «threading», la correspondance «en enfilant» serait pratique, mais son utilisation donne ici une formulation impossible. On n'enfile pas un ballon, qui d'ailleurs s'appelle ici un «ballonnet». Le traducteur va alors juxtaposer toute une série d'éléments d'information pour tenter de traduire tout le sémantisme de «threading»: il y a à la fois la notion de passage d'un fil dans quelque chose d'étroit et la notion d'entrée en force comme une vis qui creuse sa place dans le bois, par exemple. Le foisonnement n'a plus de limites si l'on décide d'écrire :

Actuellement, on traite les artères coronaires bouchées en acheminant un ballonnet dans l'artère jusqu'à l'obstruction, puis en le forçant à transpercer l'obstruction.

Il est vrai qu'il s'agit à la fois d'une progression du ballonnet dans l'artère jusqu'à l'obstruction, ou thrombus, puis d'une pénétration du ballonnet dans le thrombus, mais l'idée peut très bien être rendue par le seul verbe introduire.

To push open, pris hors contexte, ne peut être traduit en français de façon aussi économique qu'en anglais. En effet, s'il faut écrire obligatoirement «ouvrir en poussant de l'intérieur», l'argument est imparable, le français est plus long que l'anglais, et de plus de 25 pour cent. La réponse immédiate à cet argument est que la traduction d'un texte n'est pas la somme des correspondances préétablies des termes qui le composent. Un concept, pris isolément, c'est-à-dire au niveau de la langue, peut appeler en correspondance dans une autre langue tout un syntagme. Mais au niveau du texte, ce n'est pas un mot que l'on traduit, mais toute une idée. Ainsi, dans notre exemple, on peut écrire:

(...) on introduit un ballonnet puis on le gonfle pour ouvrir un passage dans l'artère.

On compte 15 mots en anglais et pas un de plus en français.

La cinquième cause est le transcodage. Le traducteur se sent contraint de traduire tous les mots par leur correspondance. En voici un exemple, extrait là encore du même rapport sur l'angioplastie par laser.

It now takes the researchers about 12 seconds after a laser pulse to analyse fluorescence from the clot.

Cette phrase a donné lieu à la traduction suivante :

Il faut maintenant environ 12 secondes aux chercheurs après une impulsion du laser pour analyser la fluorescence du caillot.

Parce qu'il lit the researchers, le traducteur écrit naturellement en français (...) aux chercheurs. Mais est-ce vraiment utile dans la phrase française? 


\section{SOLUTIONS}

Si le traducteur applique la méthode de traduction dite interprétative, il lit le texte à traduire et s'abstrait des mots pour en saisir le sens au point de pouvoir se substituer à l'auteur original pour réexprimer le texte dans une autre langue. L'appréhension totale du sens fait jaillir tout l'implicite dans l'esprit du traducteur, qui se trouve alors quasiment en situation d'expression spontanée d'une idée qu'il a pleinement assimilée et que provisoirement il a fait sienne. Le foisonnement n'est donc pas inévitable. En outre, comme le montrent les exemples ci-dessus, il n'est pas non plus souhaitable, car il nuit à la qualité et à la lisibilité de la traduction-résultat.

Pour résoudre le problème du foisonnement, nous préconisons cinq solutions. Ces solutions ne répondent pas exactement point par point aux causes énoncées ci-dessus, car certaines solutions permettent de supprimer plusieurs causes, au moins partiellement.

La première est la prise en compte de l'actualisation sémique. En effet, à la lecture d'un texte, c'est-à-dire lors de son interprétation, il convient de ne prendre en considération que la partie pertinente du sémantisme des termes, qui se trouve actualisée par le contexte, sauf bien sûr jeu de mots délibéré, auquel cas ce sont plusieurs volets de la surface conceptuelle d'un terme qui sont à retenir. Restons dans le domaine de l'angioplastie par laser.

A pioneering team of doctors in Sheffield is unblocking arteries with lasers.

Cette phrase a été traduite de la manière suivante :

Une équipe de médecins faisant œuvre de pionniers débouche maintenant les artères à l'aide de lasers.

Certes, le terme pioneering figure dans le texte original, mais est-il indispensable de parler en français de «pionnier», dont l'acception générale est «défricheur de contrées incultes» (Larousse)? Le concept de pionnier comporte plusieurs volets sémiques: aventurier, qui prend des risques, qui abandonne tout derrière lui, qui part vers l'inconnu, quelqu'un d'enthousiaste, de courageux, qui est le premier à réaliser une tâche difficile, etc. Le volet sémique qui se trouve actualisé ici est sans aucun doute celui de précurseur, de premier à tenter quelque chose de nouveau. En ne retenant que cette idée, on peut traduire le même énoncé par:

Première chirurgicale à Sheffield : des artères débouchées par laser.

Des 12 mots en anglais, il n'en reste plus que 9 en français.

La deuxième solution est la mobilisation de connaissances non linguistiques, qui permet au traducteur de bien concevoir le vouloir-dire de l'auteur original et, en conséquence, de l'exprimer clairement et avec concision, sans surcharge inutile.

The drug dissolves coronary blood clots that trigger heart attacks by blocking arteries that serve the heart.

Sachant que les coronaires sont les artères qui alimentent le muscle cardiaque, c'est-à-dire le myocarde, et que l'infarctus est la nécrose d'une partie du myocarde se trouvant privée d'apport sanguin, on peut écrire avec confiance :

Ce médicament dissout les caillots obstruant les coronaires, cause première des infarctus.

La troisième solution est la réorganisation de l'information. Dans la traduction d'un texte technique, ce qui importe, c'est de rendre dans l'autre langue le contenu du texte. Le traducteur doit allégeance à l'information, non à la structure du texte original. 
Ainsi donc, un élément d'information peut trouver logiquement sa place dans le texte traduit avant ou après le passage où il figure dans le document original. Par exemple, toujours à propos du traitement des coronaropathies :

A promising new drug that saves the lives of people who suffer heart attacks could soon be available cheaply. The drug, a human enzyme called pro-urokinase, dissolves coronary blood clots that trigger heart attacks by blocking arteries that serve the heart. The drug could undercut the cost of Eminase, an agent that dissolves clots launched last week in Britain by Beecham.

Vouloir traduire absolument «cheaply» dans la première phrase allonge considérablement celle-ci. Or, il suffit de commencer par annoncer (première phrase) la mise sur le marché prochaine d'un nouveau médicament prometteur, puis de dire (deuxième phrase), qu'il agit en dissolvant les caillots et, enfin (troisième phrase), que ce médicament ne sera pas cher (cheaply) et probablement même moins cher (could undercut the cost) que son concurrent récemment sorti des laboratoires Beecham.

En regroupant, dans le texte rendu, les deux éléments d'information qui se trouvaient dissociés dans le texte original, non seulement on limite le foisonnement, mais en outre, ce faisant, on formule l'information de façon plus logique pour un lecteur francophone et on accroît la lisibilité du document.

La quatrième solution est le transfert d' information. Un syntagme du texte original peut être porteur d'une information que le traducteur peut exprimer sous une autre forme, et à une autre occasion, dans le texte qu'il produit. Par exemple, reprenons l'extrait suivant:

Today, clogged coronary arteries are treated (...) by threading a balloon through the obstruction, then inflating it to push the artery open.

Au fond, le traitement des artères coronaires bouchées consiste à tenter d'y ouvrir un passage. Il ne serait guère élégant d'écrire qu'on traite des artères bouchées en procédant de telle ou telle manière pour les déboucher. La traduction suivante :

Aujourd'hui, pour déboucher les artères coronaires, on introduit dans le thrombus un ballonnet que l'on gonfle ensuite

permet non seulement de ne pas foisonner, mais encore de réaliser une belle économie de volume par rapport au texte original.

«Pour déboucher les artères» rend la même information que «clogged arteries are treated by

(...) to push the artery open».

Il y a transfert d'information à l'intérieur de la phrase: en chargeant de sens le début de la phrase, on peut en alléger la fin.

La cinquième solution est l'adaptation au destinataire. On ne lit pas un texte technique par hasard ou pour se distraire. Un texte technique a une mission d'information à remplir. On le lit pour s'instruire, se documenter (livres, rapports, articles, etc.) ou pour pouvoir exécuter une tâche précise (manuels, modes d'emploi, notices, etc.).

Par ailleurs, le traducteur ne traduit pas un texte technique pour l'amour de l'art, mais dans un but utilitaire. Sa traduction s'adresse donc à un public majoritairement déterminé. Si la traduction du rapport sur l'angioplastie par laser dont nous avons extrait plusieurs exemples est demandée par des spécialistes qui souhaitent connaître les résultats des expériences menées par leurs collègues étrangers, le traducteur devra se faire plus concis dans son expression, puisqu'il pourra présupposer un savoir très extensif chez les lecteurs et aussi une attente très précise de leur part. L'adoption d'un registre de langue 
plus scientifique («thrombus» pour «material blocking clogged arteries», «pontage» pour «insertion of a healthy segment of artery to bypass the blockage», par exemple) et la nonrépétition d'éléments d'information supposés connus (que la thrombose coronaire est une cause première d'infarctus, par exemple) sont des moyens de produire un texte plus concis et, en même temps et surtout, plus proche de l'attente des lecteurs.

Le lecteur d'un texte technique n'est pas nécessairement un spécialiste de la discipline, mais c'est néanmoins quelqu'un qui s'y intéresse de façon active. On peut donc, en accordant au lecteur le bénéfice du doute, considérer qu'il possède un seuil minimal d'aptitude à la compréhension du sujet traité. C'est pourquoi le fait de s'adresser à des non-spécialistes ne doit pas conduire pour autant le traducteur à surtraduire.

Dans ce cas, il adoptera un registre de langue plus familier, parlera de caillot plutôt que de thrombus et pourra préciser par exemple une fois pour toutes,

Or on sait que l'obstruction des coronaires est la cause première d'infarctus

quitte à ajouter une phrase indépendante lors de la première occurrence de la relation de cause à effet entre la présence d'un caillot obstruant une artère coronaire et l'infarctus du myocarde. Ensuite, il ne sera plus indispensable de ponctuer le texte traduit du rappel de ce type d'information.

\section{CONCLUSION}

Nous venons de dénoncer le foisonnement fortuit, véritable plaie de la traduction, qui procède le plus souvent d'un défaut de méthode et nuit à la qualité et à la lisibilité du texte traduit. Il convient également de dénoncer le foisonnement délibéré, voulu par le traducteur qui cherche ainsi à combler pour le lecteur final le manque qui résulterait du fait qu'il ne peut accéder directement au texte original. Ce n'est pas parce qu'un texte est rédigé en langue étrangère que les implicites sont nécessairement plus nombreux et transparents que dans un texte correspondant rédigé dans la langue du lecteur. C'est souvent une connaissance insuffisante de la langue étrangère et, plus souvent encore, une connaissance insuffisante du sujet traité qui fait croire au traducteur que l'énoncé en langue étrangère est plus riche que celui qu'il peut formuler dans la langue d'arrivée. En fin de compte, il est bien inutile, sous prétexte que le lecteur n'a accès qu'à un texte traduit, d'expliciter longuement l'information fournie. C'est là une tentative aussi illusoire que fallacieuse de pallier la déperdition prétendument inhérente à toute traduction. 